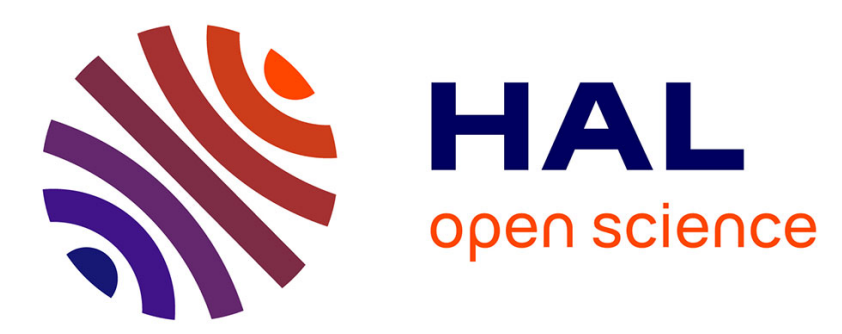

\title{
Axial-field eddy-current coupling : a 3D test problem for numerical experiments
}

Julien Fontchastagner, Thierry Lubin, Denis Netter

\section{To cite this version:}

Julien Fontchastagner, Thierry Lubin, Denis Netter. Axial-field eddy-current coupling: a 3D test problem for numerical experiments. International Journal of Numerical Modelling: Electronic Networks, Devices and Fields, 2018, 31 (2), pp.e2217. 10.1002/jnm.2217 . hal-01444185

\section{HAL Id: hal-01444185 \\ https://hal.science/hal-01444185}

Submitted on 27 Jan 2017

HAL is a multi-disciplinary open access archive for the deposit and dissemination of scientific research documents, whether they are published or not. The documents may come from teaching and research institutions in France or abroad, or from public or private research centers.
L'archive ouverte pluridisciplinaire HAL, est destinée au dépôt et à la diffusion de documents scientifiques de niveau recherche, publiés ou non, émanant des établissements d'enseignement et de recherche français ou étrangers, des laboratoires publics ou privés. 


\title{
Axial-Field Eddy-Current Coupling: a 3D Test Problem for Numerical Experiments
}

\author{
J. Fontchastagner*, T. Lubin and D. Netter \\ Université de Lorraine, GREEN, Vandœuvre-lès-Nancy, F-54500, France.
}

\begin{abstract}
SUMMARY
This paper deals with a device which is particularly relevant to test 3D quasi-static magnetic numerical computations: axial eddy-current coupling. The authors have performed several numerical FEM computations for this particular device. The eddy currents problem is solved thanks to two classical formulations based on magnetic vector and scalar potentials. Results are compared with experimental measurements made on a magnetic transmission already designed and built.
\end{abstract}

Received ...

KEY WORDS: axial magnetic couplings; quasi-static eddy currents; 3D FEM; movement.

\section{INTRODUCTION}

The purpose of this paper is to highlight a particular kind of magnetic coupling and its modelling interests. This kind of device permits to transmit an electromagnetic torque without mechanical contacts thanks to eddy currents produced by a rotating movement. Our goal is to choose the better magnetic formulation in steady-state case for several future purposes: design problems or transient simulations, for instance. First, a quick presentation of eddy-current magnetic coupling is done. In a second time, two weak formulations adapted to take into account the rotation movement are presented. Because they are widespread and well-known, the authors focus on the two classical magnetic forms, based respectively on the magnetic vector potential a and the magnetic scalar potential $\Phi$. The terms permitting to take into account the eddy current density due to the movement will then be detailed for both formulations in the particular case of steady-state condition. And finally, results provided by an efficient numerical implementation are given and compared to experimental measurements.

\section{THE ASYNCHRONOUS MAGNETIC COUPLING}

\subsection{A simple but representative $3 D$ non-linear with motion test problem}

Heavy computations are involved for a 3D formulation taking into account non-linearities and motion. To evaluate the resolution accuracy, it is convenient to refer a test problem. To take the motion into account, specific techniques (moving mesh, sliding surface,...) are usually used to avoid the remeshing of the whole domain. Nevertheless, we must use a time stepping resolution to obtain the steady state magnetic distribution. We propose a problem for which the time does not

\footnotetext{
${ }^{*}$ Correspondence to: Julien Fontchastagner, Université de Lorraine, GREEN - ENSEM, 2 avenue de la Forêt de Haye, Vandœuvre-lès-Nancy, F-54500, France. E-mail: julien.fontchastagner@univ-lorraine.fr
} 

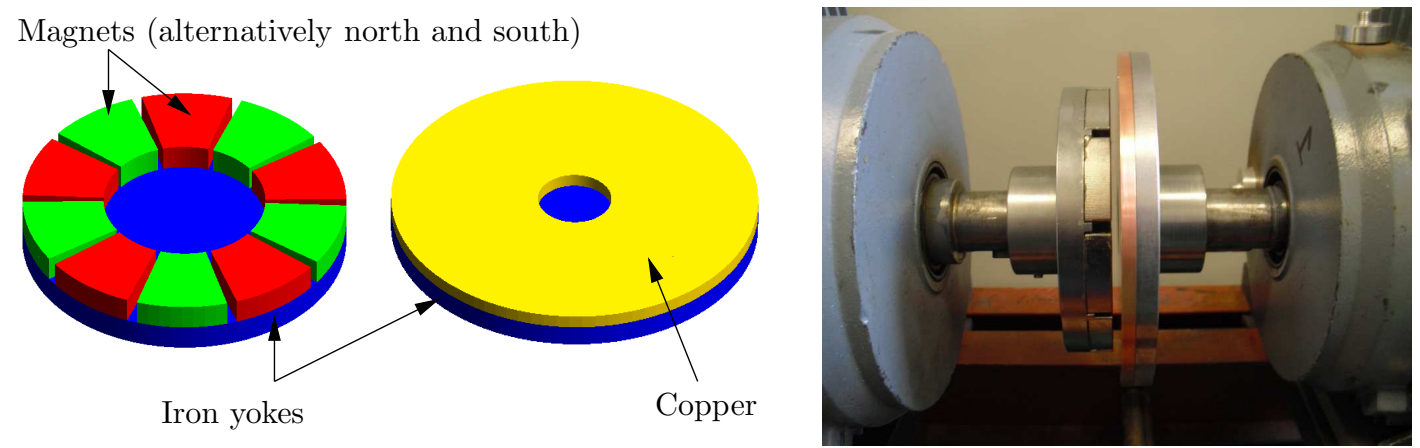

Figure 1. Schematic view of the two rotors and photography of our prototype (mounted on its test bench).

explicitely appear in the weak formulation, so that the steady state is directly calculated. Moreover, as the chosen geometry is invariant versus time, a single meshing is required.

Our model has the following characteristics:

(i) Ferromagnetic materials are non linear.

(ii) It is a real 3D problem, for which the side effects can not be neglected.

(iii) The moving part is in rotation.

\subsection{Magnetic coupling}

Magnetic couplings transmit a torque between two rotating devices without mechanical contact. Thereby, a motion can be transmitted between a mechanical source and an isolated load through an insulating wall. Another advantage is to avoid mechanical losses between the two parts. Moreover, the contactless feature does not transmit any vibrations.

This device is made of a driving part on which the engine torque is applied and a driven part on which the mechanical load is connected. The most common structure comprises permanent magnets on both moving parts. Each rotor is made of alternating north and south poles with permanent magnets glued on an iron yoke. Both radial $[1,2,3]$ or axial flux topologies $[4,5,6]$ exist. In this case, the driven and the driving parts have the same velocity. It is similar to a synchronous machine and the torque is calculated by solving a magnetostatic problem.

A less common structure is similar to an induction machine. The driving rotor remains unchanged but the driven one is replaced by a conducting plate or cylinder [7, 8, 9] glued on an iron yoke. Radial or axial structures are also possible. Moving magnets create Eddy currents in the conducting material. The torque is then due to the interaction between the field produced by the magnets and the eddy currents in the conductor. In this case, the driven and the driving parts do not turn as the same speed. Otherwise, there would be no Eddy Currents and the torque would be nil.

This device is particularly interesting in terms of numerical modelling:

(i) The geometry is purely 3D but also simple. Then, it can be meshed with structured grids. Moreover, it includes anti-periodic symmetries, allowing to reduce the computational domain to only one pole of the whole structure. An example of mesh is shown on Figure 2. It is created by the free 3D finite element grid generator Gmsh [10] (GPL licence).

(ii) The eddy currents due to movement can be computed without remeshing. If we call $N_{1}$ and $N_{2}$ the rotating speeds of respectively the driving and the driven rotors, the eddy current density is due to the slip speed $N=N_{2}-N_{1}$. The problem can be treated in considering that only the driven rotor rotates at speed $N$ (see next section 3 for details). Because of its cylindrical shape, it is useless to modify its geometry and mesh.

(iii) There are two kinds of field sources: magnets and eddy-currents. It is then more interesting to integrate them in the formulation to solve. 


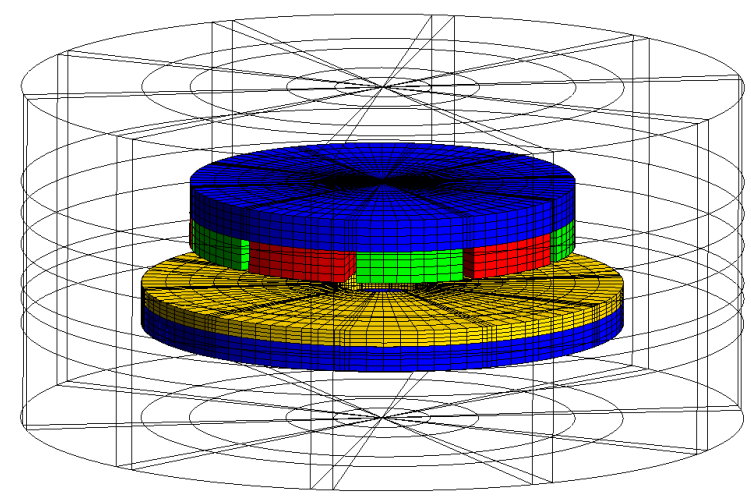

Figure 2. Example of structured mesh and domain (whole geometry).

Table I. Material properties in the different domains

\begin{tabular}{c|c|c|c|c}
\hline domain & material name & relative permeability $\mu_{r}$ & conductivity $\sigma$ & remanence $B_{r}$ \\
\hline$\Omega_{y m}, \Omega_{y c}$ & M1010 steel & linear: 8000, else $: b(h)$ curve & $7 \mathrm{MS} \cdot \mathrm{m}^{-1}$ & - \\
$\Omega_{m}$ & NdFeB & 1 & - & $1.25 \mathrm{~T}$ \\
$\Omega_{c}$ & copper & 1 & $57 \mathrm{MS} \cdot \mathrm{m}^{-1}$ & - \\
\hline
\end{tabular}

(iv) Its simplicity permits to easily build prototypes at an affordable price. Our prototype is shown in Figure 1 and we have used it to connect two DC machines: a motor and its load. We can cheaply obtain experimental measurements to validate our computations.

\section{STEADY-STATE MAGNETIC MODELLING}

\subsection{Problem definition}

In steady-state, the driving rotor rotates at speed $N_{1}$ and the driven one at speed $N_{2}$. If the reference frame $\left(\mathrm{O}, \mathbf{u}_{\mathbf{x}}, \mathbf{u}_{\mathbf{y}}, \mathbf{u}_{\mathbf{z}}\right)$ link to the first rotor is chosen, we can consider that only the second rotor (with the conductor part) rotates at speed $N=N_{2}-N_{1}$. All the other parts are motionless. $N$ is the slip speed of the coupling, the $z$ axis is considered to be the rotation axis, then the rotation vector is $\mathbf{N}=N \mathbf{u}_{\mathbf{z}}$.

We define all the domains and boundaries which constitute the geometry. All of them are displayed on Figure 3 (a), for one pole:

- $\Omega_{m}$ : magnets,

- $\Omega_{y m}$ : yoke of the magnets side,

- $\Omega_{c}$ : copper ring,

- $\Omega_{y c}$ : yoke of the copper side,

- $\Omega_{a}$ : air around the two rotors,

- $\Gamma_{d}$ : domain boundaries (top, bottom, inner and outer surfaces),

- $\Gamma_{a}$ : left and right surfaces with anti-periodic condition.

The corresponding material parameters are given in Table I. In the case of a non-linear magnetic relationship, the $b(h)$ curve used for M1010 steel is given by Figure 3 (b). Magnets remanence is defined by: $\mathbf{b}_{\mathbf{r}}= \pm B_{r} \mathbf{u}_{\mathbf{z}}$. 


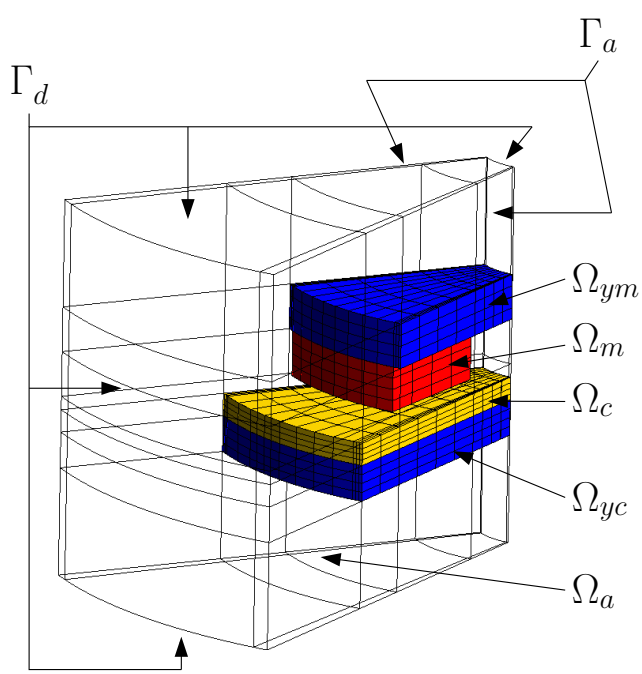

(a)

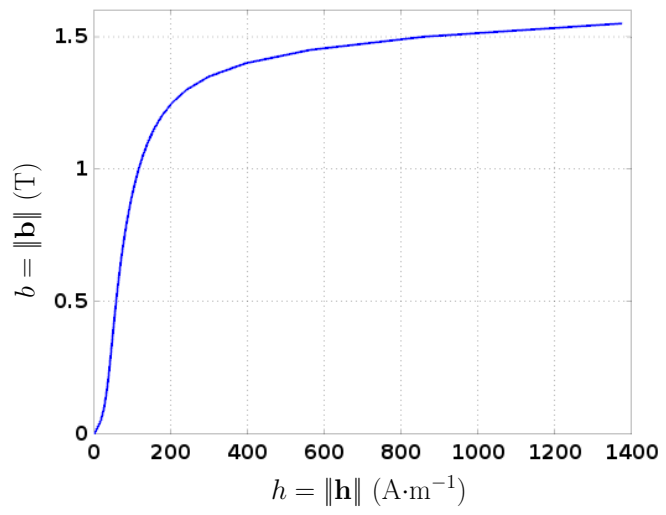

(b)

Figure 3. (a): Definition of domains and boundaries (1 pole); and (b): $b(h)$ curve used for M1010 steel.

Under the previous hypotheses, if the position vector is called $\mathbf{p}$ (i.e. $\mathbf{p}=(x, y, z)$ ), the speed at $\mathbf{p}$ is given by:

$$
\mathbf{v}=\mathbf{N} \times \mathbf{p}
$$

The partial time derivative of the flux density is given by:

$$
\partial_{t} \mathbf{b}=-\operatorname{curl}(\mathbf{v} \times \mathbf{b})
$$

And the Maxwell-Faraday equation becomes:

$$
\operatorname{curl}(\mathbf{e}+\mathbf{v} \times \mathbf{b})=\operatorname{curl}(\mathbf{e}+(\mathbf{N} \times \mathbf{p}) \times \mathbf{b})=0
$$

The two classical formulations of magnetostatics, including terms due to movement, can then be applied to the problem.

\subsection{Quasi-static magnetic formulations}

3.2.1. Magnetic vector potential (a, $v$ ) formulation Equation (3) means that we can use the modified Ohm's law in our particular case. Introducing the magnetic vector potential a and scalar electric potential $v$, and using (1), the eddy current density in conductors is given by:

$$
\mathbf{j}_{\mathbf{e}}=\sigma(-\operatorname{grad} v+(\mathbf{N} \times \mathbf{p}) \times \operatorname{curl} \mathbf{a})
$$

The weak form of the governing equation can be established. The total domain $\Omega$, the whole conducting domain $\Omega_{c c}$ and its boundaries $\Gamma_{c}$ are defined by:

$$
\begin{aligned}
\Omega & =\Omega_{m} \cup \Omega_{y m} \cup \Omega_{c} \cup \Omega_{y c} \cup \Omega_{a} \\
\Omega_{c c} & =\Omega_{c} \cup \Omega_{y c} \\
\Gamma_{c} & =\partial \Omega_{c c}
\end{aligned}
$$

The complete variational $(\mathbf{a}, v)$ formulation is then given by:

$$
\left\{\begin{array}{c}
\left(\mu^{-1} \operatorname{curl} \mathbf{a}, \operatorname{curl} \mathbf{a}^{\prime}\right)_{\Omega}-\left(\mu^{-1} \mathbf{b}_{\mathbf{r}}, \operatorname{curl} \mathbf{a}^{\prime}\right)_{\Omega_{m}}+\left(\sigma \operatorname{grad} v, \mathbf{a}^{\prime}\right)_{\Omega_{c c}} \\
-\left(\sigma(\mathbf{N} \times \mathbf{p}) \times \operatorname{curl} \mathbf{a}, \mathbf{a}^{\prime}\right)_{\Omega_{c c}}=0 \\
\left(\operatorname{grad} v, \operatorname{grad} v^{\prime}\right)_{\Omega_{c c}}-\left(\sigma(\mathbf{N} \times \mathbf{p}) \times \operatorname{curl} \mathbf{a}, \operatorname{grad} v^{\prime}\right)_{\Omega_{c c}}=0 \\
\forall \mathbf{a}^{\prime} \in \boldsymbol{H}_{0}(\operatorname{curl} ; \Omega), \forall v^{\prime} \in H_{0}^{1}\left(\Omega_{c c}\right)
\end{array}\right.
$$


3.2.2. Magnetic scalar potential $\left(\mathbf{h}_{\mathbf{e}}, \phi\right)$ formulation Thanks to $\operatorname{div} \mathbf{j}=0$, a source field $\mathbf{h}_{\mathbf{e}}$ producing eddy currents in conductors can be defined by:

$$
\mathrm{j}_{\mathrm{e}}=\operatorname{curl}_{\mathrm{e}}
$$

Then, introducing the magnetic scalar potential $\phi$, the magnetic field $\mathbf{h}$ becomes:

$$
\mathbf{h}=\mathbf{h}_{\mathbf{e}}-\operatorname{grad} \phi
$$

Using (3) and Maxwell-Thomson equation, the complete variational $\left(\mathbf{h}_{\mathbf{e}}, \phi\right)$ formulation can be expressed by:

$$
\left\{\begin{array}{c}
\left(\mu \mathbf{h}_{\mathbf{e}}, \operatorname{grad} \phi^{\prime}\right)_{\Omega_{c c}}-\left(\mu \operatorname{grad} \phi, \operatorname{grad} \phi^{\prime}\right)_{\Omega}+\left(\mathbf{b}_{\mathbf{r}}, \operatorname{grad} \phi^{\prime}\right)_{\Omega_{m}}=0 \\
\left(\sigma^{-1} \operatorname{curl}_{\mathbf{e}}, \operatorname{curl}_{\mathbf{e}}\right)_{\Omega_{c c}}-\left(\mu(\mathbf{N} \times \mathbf{p}) \times \mathbf{h}_{\mathbf{e}}, \operatorname{curl}_{\mathbf{e}}\right)_{\Omega_{c c}} \\
+\left(\mu(\mathbf{N} \times \mathbf{p}) \times \operatorname{grad} \phi, \operatorname{curl}_{\left.\mathbf{h}_{\mathbf{e}}{ }^{\prime}\right)_{\Omega_{c c}}}=0\right. \\
\forall \phi^{\prime} \in H_{0}^{1}(\Omega), \forall \mathbf{h}_{\mathbf{e}}{ }^{\prime} \in \boldsymbol{H}_{0}\left(\operatorname{curl} ; \Omega_{c c}\right)
\end{array}\right.
$$

\subsection{Post-processing quantities computations}

3.3.1. Joule losses Knowing a and $v$, or $\mathbf{h}_{\mathbf{e}}$, the eddy current density in $\Omega_{c c}$ can be computed by (4) or (7). Then, the total Joule losses can be evaluated by:

$$
P_{J}= \begin{cases}\int_{\Omega_{c c}} \sigma\|\operatorname{grad} v-(\mathbf{N} \times \mathbf{p}) \times \operatorname{curl} \mathbf{a}\|^{2} d \Omega & \text { if }(\mathbf{a}, v) \text { form. } \\ \int_{\Omega_{c c}} \sigma^{-1}\left\|\mathbf{c u r l} \mathbf{h}_{\mathbf{e}}\right\|^{2} d \Omega & \text { if }\left(\mathbf{h}_{\mathbf{e}}, \phi\right) \text { form. }\end{cases}
$$

3.3.2. Electromagnetic torque There are three ways to compute the EM torque transmitted by the coupling. Our opinion is that one of the following method is not better than an other. But the fact to have several means to compute the same quantity is really interesting. Indeed, if the obtained results are similar, it permits to validate our problem statement and more especially the used mesh. The three proposed methods are :

(i) By the Joule Losses

In steady-state, a power balance between the driving part $\left(P_{1}\right)$ and the driven one $\left(P_{2}\right)$ gives:

$$
P_{1}=T \cdot N_{1}=P_{2}+P_{J}=T \cdot N_{2}+P_{J}
$$

where $T$ is the electromagnetic torque which is equal, in our particular case, to the useful transmitted mechanical torque (since there is no mechanical losses in such a magnetic torque transmission). Then we can deduced the expression $T_{J L}$ of the torque based on the Joule losses and the slip speed $N$ by:

$$
T_{J L}=-\frac{P_{J}}{N}
$$

(ii) By the Laplace forces

The Laplace forces in the $\Omega_{c c}$ conducting domain can be computed from $\mathbf{a}$ and $v$, or $\mathbf{h}_{\mathbf{e}}$ and $\phi$. And then, the EM torque can be directly evaluated by:

$$
T_{L F}=\left\{\begin{array}{l}
\int_{\Omega_{c c}}[\mathbf{p} \times(\sigma[-\operatorname{grad} v+(\mathbf{N} \times \mathbf{p}) \times \operatorname{curl} \mathbf{a}] \times \operatorname{curl} \mathbf{a})] \cdot \mathbf{u}_{\mathbf{z}} d \Omega \\
\int_{\Omega_{c c}}\left[\mathbf{p} \times\left(\operatorname{curl} \mathbf{h}_{\mathbf{e}} \times\left[\mu\left(\mathbf{h}_{\mathbf{e}}-\operatorname{grad} \phi\right)\right]\right)\right] \cdot \mathbf{u}_{\mathbf{z}} d \Omega
\end{array}\right.
$$


(iii) By the Maxwell stress tensor

Knowing the magnetic field $\mathbf{h}$ and flux density $\mathbf{b}$ in the airgap, we can applied the Maxwell stress tensor on a cylindrical closed surface containing the driven rotor. If the radius of this cylinder is extended until to the domain boundaries, the bounds of the surface integral given the torque can be limited to a plain disc in the airgap. A method to reduce numerical noise is to perform an average of this surface integral by introducing a volume integral of the tensor in the whole cylindrical air domain between the two rotors called $\Omega_{g}$. This technique had been successfully applied in [6]. Then, in this case, the EM torque is provided by:

$$
T_{M T}=\left\{\begin{array}{l}
\frac{1}{\mu_{0} g} \int_{\Omega_{g}}\left[y\left(\operatorname{curl} \mathbf{a} \cdot \mathbf{u}_{\mathbf{x}}\right)-x\left(\operatorname{curl} \mathbf{a} \cdot \mathbf{u}_{\mathbf{y}}\right)\right]\left(\operatorname{curl} \mathbf{a} \cdot \mathbf{u}_{\mathbf{z}}\right) d \Omega \\
\frac{\mu_{0}}{g} \int_{\Omega_{g}}\left[y\left(\operatorname{grad} \phi \cdot \mathbf{u}_{\mathbf{x}}\right)-x\left(\operatorname{grad} \phi \cdot \mathbf{u}_{\mathbf{y}}\right)\right]\left(\operatorname{grad} \phi \cdot \mathbf{u}_{\mathbf{z}}\right) d \Omega
\end{array}\right.
$$

where $g$ is the airgap thickness.

\section{NUMERICAL EXPERIMENTS}

\subsection{Implementation}

The both previous formulations had been implemented using the free and open source finite element solver GetDP [11]:

(i) a and $v$ are respectively approximated in an edge element space over $\Omega$ and a nodal element space over $\Omega_{c c}$. The uniqueness of $\mathbf{a}$ is insured thanks to a spanning tree gauge [12]. Dirichlet condition upon a is applied on $\Gamma_{d}$.

(ii) The $\left(\mathbf{h}_{\mathbf{e}}, \phi\right)$ formulation is the dual case: we use respectively an edge element space over $\Omega_{c c}$ and a nodal element space over $\Omega$. Cause of the simple shape of $\Omega_{c c}$, no gauge or cut is needed for $\mathbf{h}_{\mathbf{e}}$ [13]. Dirichlet condition upon $\mathbf{h}_{\mathbf{e}}$ is applied on $\Gamma_{c}$ (boundary defined by (5)).

For complementary theoretical aspects around the two formulations and their implementation, see $[14,15,16]$. For better performances, the GetDP solver and its third parties (PETSc [17] and MUMPS [18]) had been locally recompiled on the workstation used for tests. This one is a simple laptop (with Intel Core i7-5500U @ 2.4 GHz) running a Debian GNU/Linux system. The created binary is a sequential version with multithreading support: $2 \times 2$ threads are used in tests below.

\subsection{Linear case}

First, we solve a simpler linear problem with $\mu_{r}=8000$ that corresponds to the linear part of the $b(h)$ constitutive law (Figure $3(\mathrm{~b})$ ). The purpose of this test case is to validate the accounting of the anti-periodic conditions when the geometry is reduced to one pole.

Problems corresponding to both geometries with both formulations are solved and the results are given in Table II. The used geometric parameters are those of our prototype, the slip speed is fixed to $N=-\frac{2 \pi}{60} \cdot 140 \mathrm{rad} \cdot \mathrm{s}^{-1}$. The given CPU times correspond to the total time needed to solve the problem (geometry, meshing, resolution and post-processing). The current density in the copper part for the whole geometry is also shown in Figure 4, for both formulations.

All our results are in good agreement with each others. The values computed by the $(\mathbf{a}, v)$ formulation are just a bit greater than those issued from $\left(\mathbf{h}_{\mathbf{e}}, \phi\right)$. Anti-periodic conditions have a slightly influence on the first form, and absolutely none on the $\left(\mathbf{h}_{\mathbf{e}}, \phi\right)$ one. It can be explained by the fact that it is not exactly the same domain for the whole system and for the one pole case. Indeed, because of geometric facilities, in the anti-periodic case, the small cylindrical air volume at the centre of the geometry (and containing the $z$ axis) had been replace by a surface included in $\Gamma_{d}$. Then the mesh and the gauge condition are not exactly the same for both models. It implies some differences on the values of $\mathbf{a}$, which produce the small gaps on global quantities. This phenomenon 
Table II. Comparison of results (linear case: $\mu_{r}=8000,-140 \mathrm{rpm}$ )

\begin{tabular}{|c|c|c|c|c|}
\hline Formulation & \multicolumn{2}{|c|}{$(\mathbf{a}, v)$} & \multicolumn{2}{|c|}{$\left(\mathbf{h}_{\mathbf{e}}, \phi\right)$} \\
\hline $\begin{array}{l}\text { Geometry } \\
\text { Dofs }\end{array}$ & $\begin{array}{c}\text { complete } \\
208,96\end{array}$ & $\begin{array}{r}1 \text { pole } \\
24,820\end{array}$ & $\begin{array}{c}\text { complete } \\
162,120\end{array}$ & $\begin{array}{c}1 \text { pole } \\
19,940\end{array}$ \\
\hline$P_{J}(\mathrm{~W})$ & 126.4 & 128.8 & 121.5 & 121.5 \\
\hline $\begin{array}{l}T_{J L}(\mathrm{~N} \cdot \mathrm{m}) \\
T_{L F}(\mathrm{~N} \cdot \mathrm{m}) \\
T_{M T}(\mathrm{~N} \cdot \mathrm{m})\end{array}$ & $\begin{array}{l}8.62 \\
8.73 \\
8.63\end{array}$ & $\begin{array}{l}8.79 \\
8.90 \\
8.75\end{array}$ & $\begin{array}{l}8.29 \\
8.29 \\
8.30\end{array}$ & $\begin{array}{l}8.29 \\
8.29 \\
8.30\end{array}$ \\
\hline $\begin{array}{c}\text { CPU time (sec) } \\
\text { RAM (MB) }\end{array}$ & $\begin{array}{l}143.7 \\
2,161\end{array}$ & $\begin{array}{l}9.53 \\
200\end{array}$ & $\begin{array}{l}138.3 \\
2,032\end{array}$ & $\begin{array}{l}6.05 \\
198\end{array}$ \\
\hline
\end{tabular}

$\mathbf{j}_{\mathbf{e}}=\sigma(-\operatorname{grad} v+(\mathbf{N} \times \mathbf{p}) \times \operatorname{curl} \mathbf{a})$
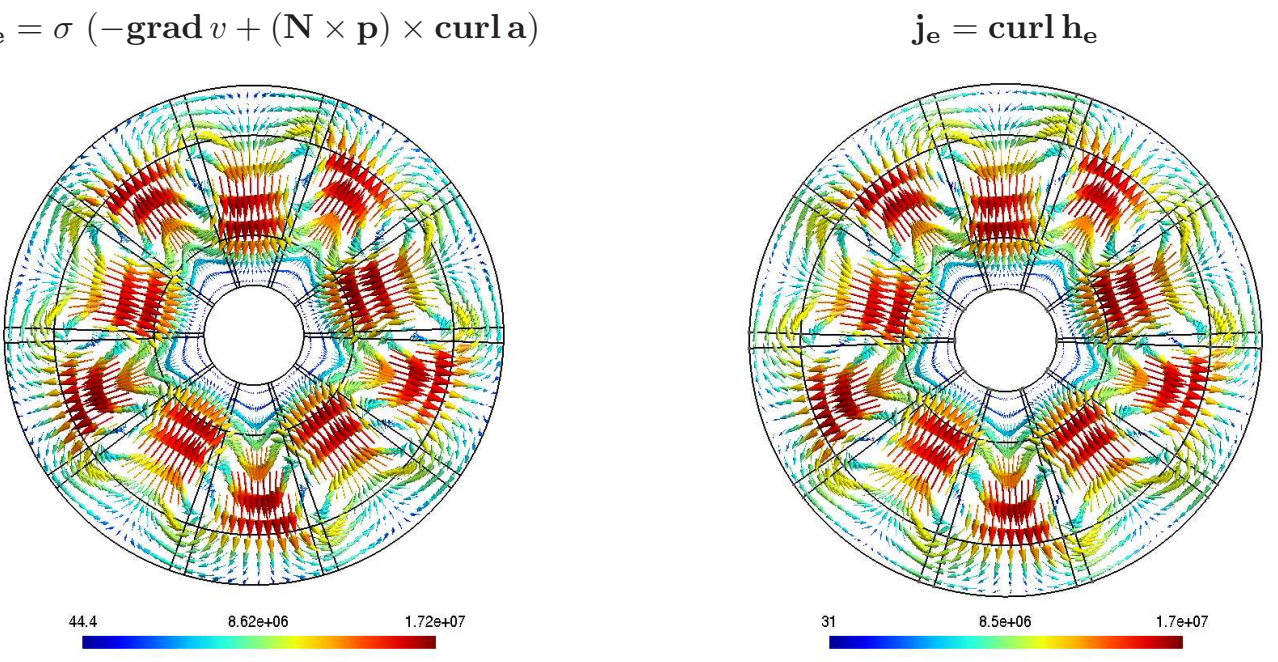

Figure 4. Eddy current density computed by the two formulations (linear case).

do not occur for the $\left(\mathbf{h}_{\mathbf{e}}, \phi\right)$ formulation because the copper domain remains the same in the two cases (complete or one pole geometries). Nevertheless, we can considered that the problem reduced to one pole is solved correctly. Moreover, we can verify that the computed torques are positive, it is normal since its sign is obviously the opposite of the one of the slip speed, according to the Lenz's law. In front of the CPU time savings (more than ratio 10), only the one pole case will be considered in the following.

As shown in Figure 5, the flux density is greater than $1.6 \mathrm{~T}$ (saturated value of the $b(h)$ curve). As a result, we must solve a non-linear problem.

\subsection{Non-linear case}

Now, the non-linear $b(h)$ curve given in Figure 3 (b) is used for the $\Omega_{y m}$ and $\Omega_{y c}$ domains. Several non-linear solvers had been tested and, for our problems, the best performances were obtained with:

(i) the PETSc SNES solver with its default options for the (a, $v)$ formulation;

(ii) our own fixed point method (implemented using the GetDP scripting facilities) for the $\left(\mathbf{h}_{\mathbf{e}}, \phi\right)$ formulation. This problem is a bit hard to converge because of $\Omega_{y c}$ domain which is both a conducting and magnetic material. The permeability $\mu$ in this domain depends on the both unknowns $\mathbf{h}_{\mathbf{e}}$ and $\phi$, whereas in the $(\mathbf{a}, v)$ case, it depends only on $\mathbf{a}$.

The same stopping criterion is used for both resolutions: iterations are stopped when the residual norm is less than $10^{-3}$. To accelerate even more the convergence time, the first iteration of the nonlinear problem is initialised with the solution of a linear case. This classical method permits to avoid 2 or 3 iterations during the non-linear resolution. 


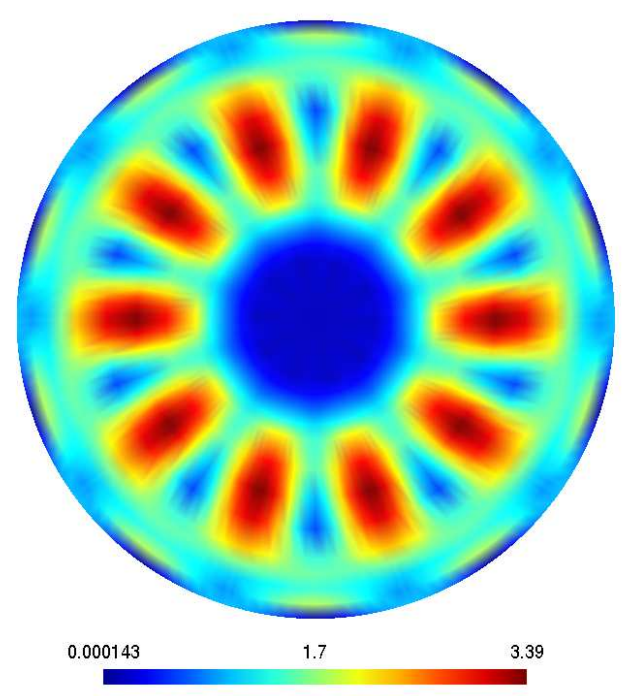

Figure 5. Flux density norm at the copper/yoke interface (linear case).

Figure 6 shows flux density maps for the linear and the non-linear cases. As expected, flux density maximum value reaches around 1.6 Tesla for the non-linear case, proving that the yoke is locally strongly saturated.

Table III compares torques (computed by the three methods detailed in the previous section) and Joule losses for linear and non-linear cases. Results highlights the fact that torque values given by the three methods are really close. CPU times are very small. They include all the resolution process, from geometry to post-processing, and are then truly satisfactory.

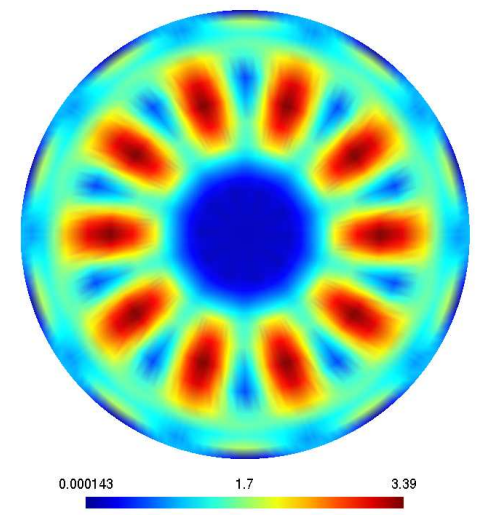

Linear case (same as in Figure 5)

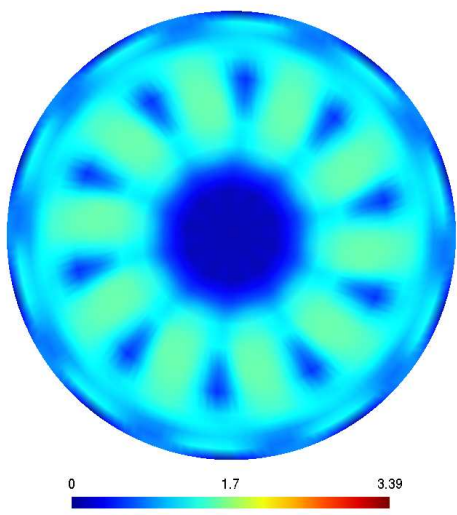

Non-linear case

Figure 6. Norm of flux density at the copper/yoke interface $(N=-140 \mathrm{rpm}$, $g=3 \mathrm{~mm},(\mathbf{a}, v)$ formulation, and same scale).

Torque values are really close to those computed for the linear case and magnetic saturation does not seem to affect these global quantities. This can be explained by Figure 7, which displays the flux density map in the middle of the airgap for both linear and non-linear cases. It appears that the flux density is exactly the same in these two cases. Then, the magnetic saturation is only located at the copper/iron interface and it does not impact the flux density in the airgap and copper regions. So, the eddy currents are almost the same. Saturation should affect the torque value if the yoke was entirely saturated. 
Table III. Comparison of results (linear vs non-linear case) for $N=-140 \mathrm{rpm}$

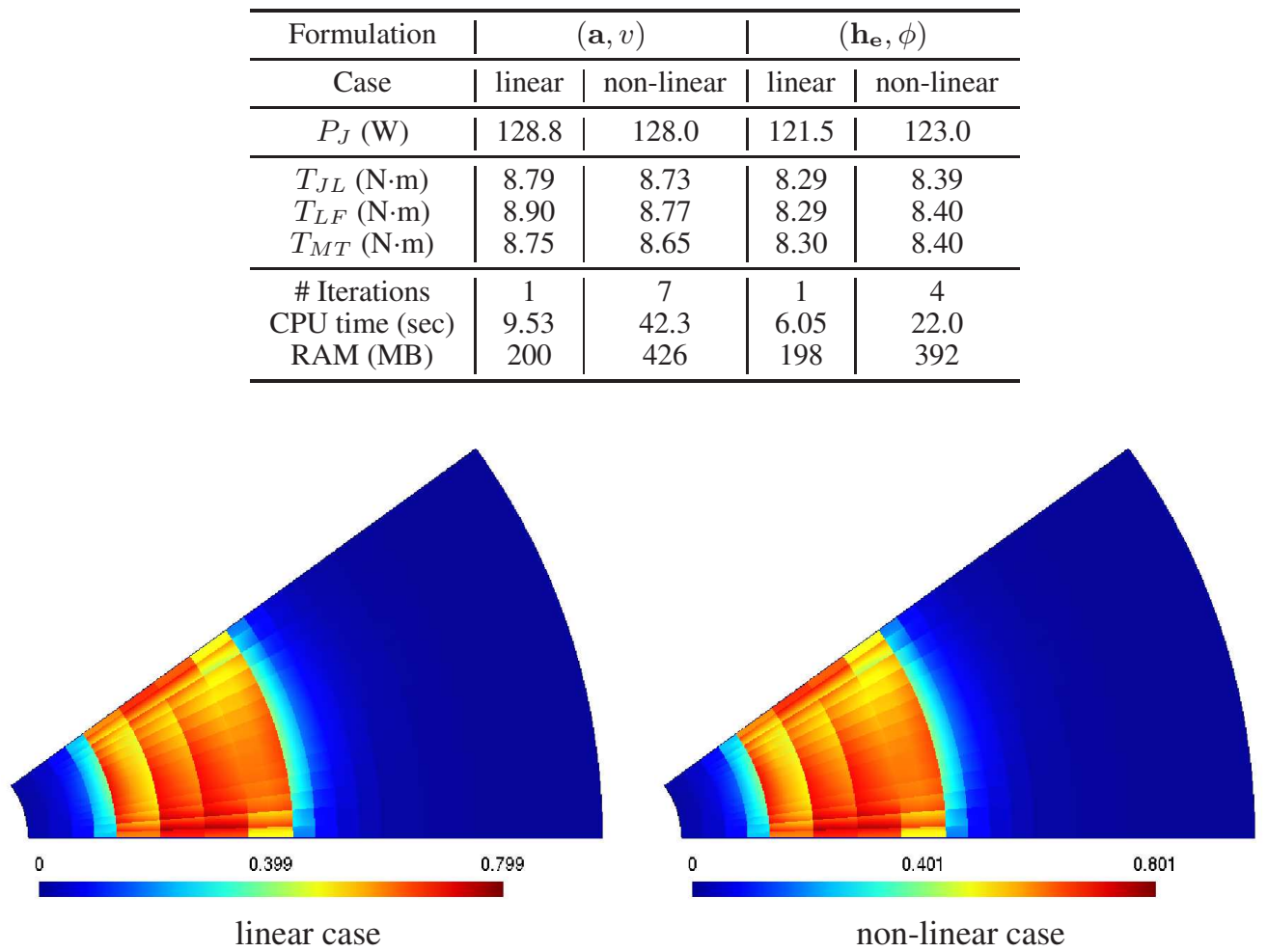

Figure 7. Flux density map at the centre of the airgap, in both linear and non-linear cases (one pole).

In order to validate our results on a wide range of points, a comparison between experimental values and our models is performed for several airgap thicknesses $(g=3,5$ and $7 \mathrm{~mm})$, and for several slip speed values. The chosen bounds correspond to the limitations of the developed test bench as shown on Figure 1. The obtained results are given on Figure 8, where the absolute values of slip speed are used for clarifing the plots. For the both formulation, the results point out a good agreement between measurements and computations.

Thus, at the question: "Which magnetic formulation to choose?", the answer should be: "It depends". Both have real advantages. If we want to emphasise accuracy, the $(\mathbf{a}, v)$ seems to be more convenient. Indeed, the results obtained with it are a bit more close to experiments. In a near future, it will be privileged for performing a complete transient simulation of the device with kinematic and thermal couplings. But, if we want to deal with a design process based only on the steadystate behaviour, the $\left(\mathbf{h}_{\mathbf{e}}, \phi\right)$ formulation should be the best choice. Since it is the faster one, and computed values are slightly underestimate the real torque values (good for an inequality constraint satisfaction), it will be really suitable for implementation with a metaheuristic optimisation method such as any population algorithm.

\section{CONCLUSION}

In this paper, the authors present a simple but interesting magnetic device: an axial field eddycurrent coupling. A study on the steady-state behaviour of this particular application is performed. It is based on the two classical weak forms suited to account eddy-current density due to movement. An efficient implementation of both formulations $(\mathbf{a}, v)$ and $\left(\mathbf{h}_{\mathbf{e}}, \phi\right)$ provides good results in terms of rapidity and accuracy compared to experimental values. Finally, a choice criterion between the two methods is given. In a future work, both formulations will be used. First, the authors want 

$(\mathbf{a}, v)$ formulation

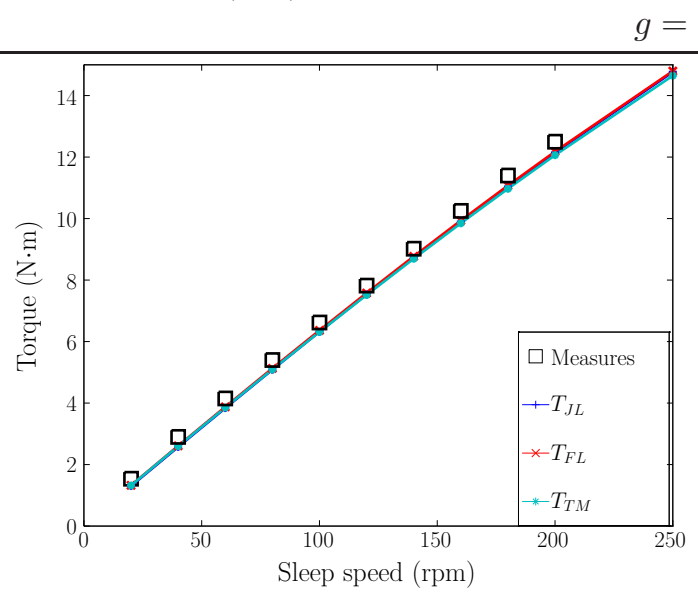

$\left(\mathbf{h}_{\mathbf{e}}, \phi\right)$ formulation $g=3 \mathrm{~mm}$
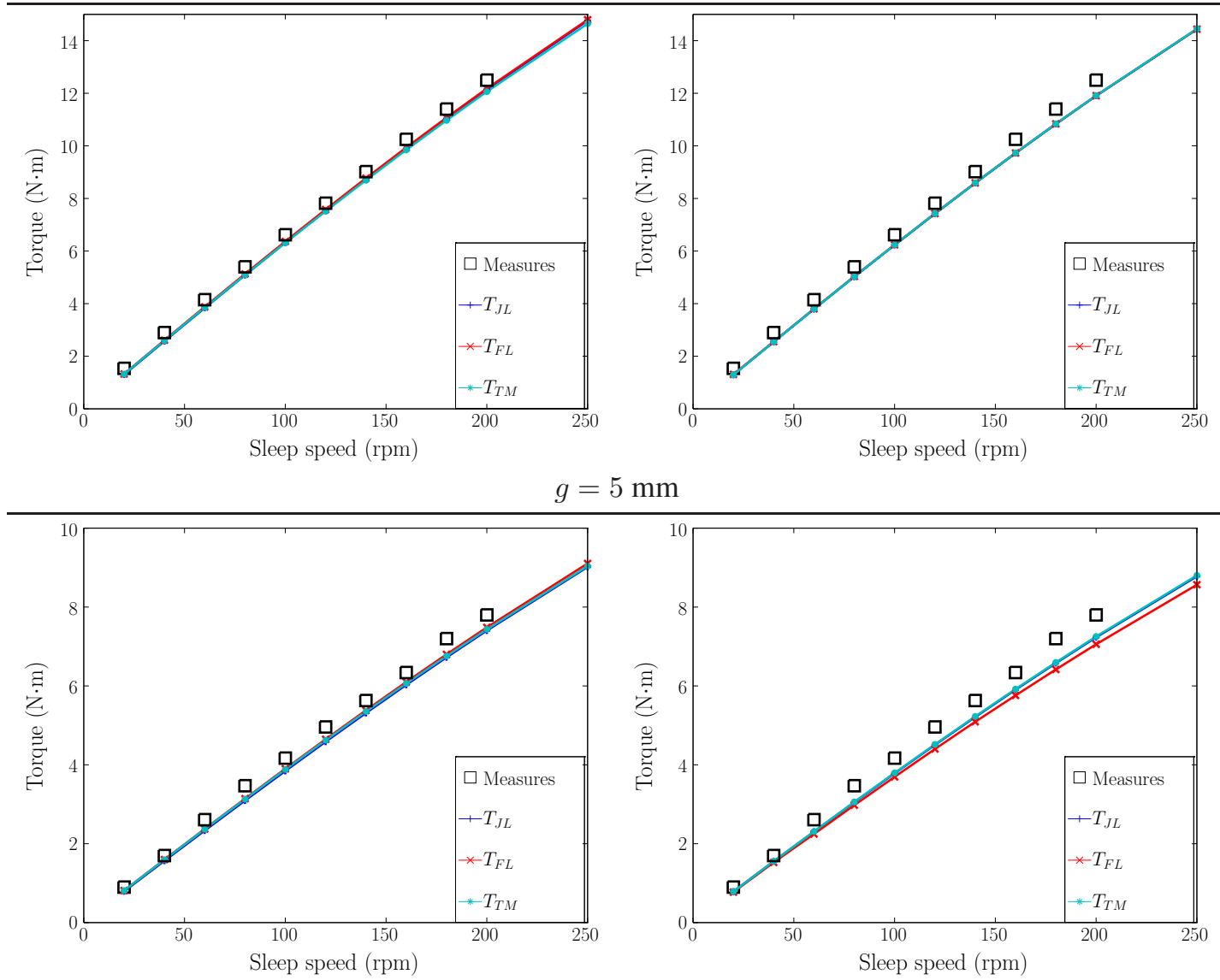

$g=7 \mathrm{~mm}$
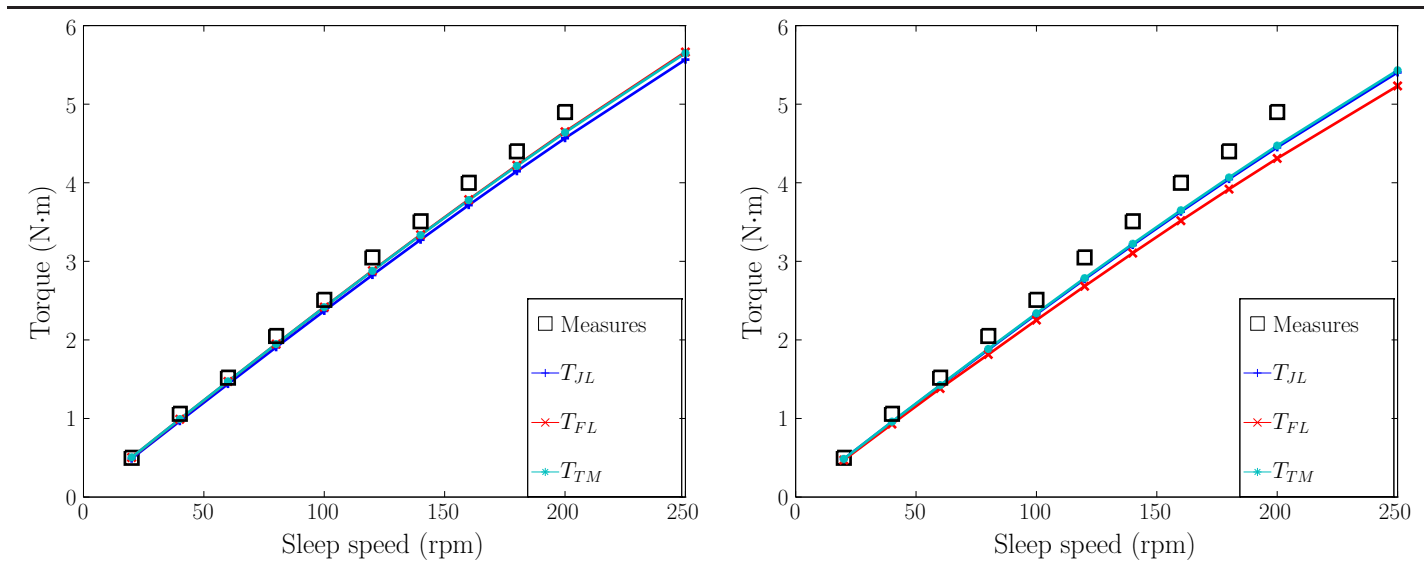

Figure 8. Torque vs. slip speed characteristics (left: $(\mathbf{a}, v)$, right: $\left(\mathbf{h}_{\mathbf{e}}, \phi\right)$ ).

to perform a complete multi-physics simulation of an axial field eddy-current coupling, including kinematics and thermal effects. Then, they will used the $(\mathbf{a}, v)$ formulation because it seems more close to experimental values. In a second time, they also want to solve the design problem of such devices by optimisation techniques. In that case, they will used the $\left(\mathbf{h}_{\mathbf{e}}, \phi\right)$ because it provides a good torque under-evaluation in a really short time. 


\section{ACKNOWLEDGEMENTS}

The work presented in this paper had been performed using only free and open source software. So, the authors thank all the contributors of these tools. More especially, they strongly acknowledge the Gmsh and GetDP developers for sharing their work with the community.

\section{REFERENCES}

1. Yonnet JP, Hemmerlin S, Rulliere E, Lemarquand G. Analytical calculation of permanent magnet couplings. IEEE Trans. Magn. Nov 1993; 29(6):2932-2934.

2. Charpentier JF, Lemarquand G. Optimal design of cylindrical air-gap synchronous permanent magnet couplings. IEEE Trans.Magn. Mar 1999; 35(2):1037-1046.

3. Fontchastagner J, Lefèvre Y, Messine F. Some co-axial magnetic couplings designed using an analytical model and an exact global optimization code. IEEE Trans. Magn. Mar 2009; 45(3):1458-1461.

4. Wu W, Lovatt HC, Dunlop JC. Analysis and design optimisation of magnetic couplings using 3D finite element modelling. IEEE Trans. Magn. Sept 1997; 33(5):4083-4085.

5. Lubin T, Mezani S, Rezzoug A. Simple analytical expressions for the force and torque of axial magnetic couplings. IEEE Trans. Energy. Convers. Jun 2012; 27(2):536-546.

6. Fontchastagner J, Lubin T, Messine F, Mezani S. Efficient design using successive analytical subproblems method: Application to axial magnetic couplings. IEEE Trans. Magn. Mar 2015; 51(3):1-4.

7. Davis EJ, Wright MT, Johnson RC. Transient performance of eddy-current couplings. Proc. IEE Oct 1975; 122(10): $1128-1136$

8. Canova A, B V. Analytical modeling of rotating eddy-current couplers. IEEE Trans. Magn. Janv 2005; 41(1):24-35.

9. Lubin T, Rezzoug A. Steady-state and transient performance of axial-field eddy-current coupling. IEEE Trans. Ind. Electron Apr 2015; 62(4):2287-2296.

10. Geuzaine C, Remacle JF. Gmsh: a three-dimensional finite element mesh generator with built-in pre- and postprocessing facilities. Int. J. Numer. Meth. Engng 2009; 79(11):1309-1331.

11. Geuzaine C. GetDP: a general finite-element solver for the de Rham complex. PAMM Volume 7 Issue 1. Special Issue: Sixth International Congress on Industrial Applied Mathematics (ICIAM07) and GAMM Annual Meeting, Zürich 2007, vol. 7, Wiley, 2008; $1010603-1010604$.

12. Dular P, Nicolet A, Genon A, Legros W. A discrete sequence associated with mixed finite elements and its gauge condition for vector potentials. IEEE Trans. Magn. May 1995; 31(3):1356-1359.

13. Dular P, Henrotte F, Robert F, Genon A, Legros W. A generalized source magnetic field calculation method for inductors of any shap. IEEE Trans. Magn. Mar 1997; 33(2):1398-1401.

14. Bossavit A, Verite J. The "TRIFOU" code: Solving the 3-D eddy-currents problem by using H as state variable. IEEE Trans. Magn. Nov 1983; 19(6):2465-2470.

15. Bossavit A. Two dual formulations of the 3D eddycurrents problem. COMPEL 1985; 4(2):103-116.

16. Ren Z, Razek A. Computation of 3-D electromagnetic field using differential forms based elements and dual formulations. International Journal of Numerical Modelling: Electronic Networks, Devices and Fields 1996; 9(12):81-98.

17. Balay S, Abhyankar S, Adams MF, Brown J, Brune P, Buschelman K, Dalcin L, Eijkhout V, Gropp WD, Kaushik D, et al.. PETSc Web page. http://www. mcs.anl.gov/petsc 2015.

18. Amestoy PR, Duff IS, Koster J, L'Excellent JY. A fully asynchronous multifrontal solver using distributed dynamic scheduling. SIAM Journal on Matrix Analysis and Applications 2001; 23(1):15-41. 